\title{
Performance assessment of the single photon emission microscope: high spatial resolution SPECT imaging of small animal organs
}

\author{
J. Mejia ${ }^{1}$, M.A. Reis ${ }^{1,2}$, A.C.C. Miranda ${ }^{1}$, I.R. Batista ${ }^{1,2}$, M.R.F. Barboza ${ }^{1}$, M.C. Shih ${ }^{1}$, G. Fu ${ }^{3}$ \\ C.T. Chen ${ }^{4}$, L.J. Meng ${ }^{5}$, R.A. Bressan ${ }^{1,2}$ and E. Amaro Jr. ${ }^{1}$ \\ ${ }^{1}$ Hospital Israelita Albert Einstein, Instituto do Cérebro, São Paulo, SP, Brasil \\ ${ }^{2}$ Laboratório Interdisciplinar de Neurociências Clínicas, Departamento de Psiquiatria, \\ Universidade Federal de São Paulo, São Paulo, SP, Brasil \\ ${ }^{3}$ GE Global Research, Schenectady, NY, USA \\ ${ }^{4}$ Department of Radiology, University of Chicago, Chicago, IL, USA \\ ${ }^{5}$ Department of Nuclear, Plasma and Radiological Engineering, University of Illinois, Urbana-Champaign, IL, USA
}

\begin{abstract}
The single photon emission microscope (SPEM) is an instrument developed to obtain high spatial resolution single photon emission computed tomography (SPECT) images of small structures inside the mouse brain. SPEM consists of two independent imaging devices, which combine a multipinhole collimator, a high-resolution, thallium-doped cesium iodide [CsI(TI)] columnar scintillator, a demagnifying/intensifier tube, and an electron-multiplying charge-coupling device (CCD). Collimators have 300- and 450- $\mu \mathrm{m}$ diameter pinholes on tungsten slabs, in hexagonal arrays of 19 and 7 holes. Projection data are acquired in a photon-counting strategy, where CCD frames are stored at 50 frames per second, with a radius of rotation of $35 \mathrm{~mm}$ and magnification factor of one. The image reconstruction software tool is based on the maximum likelihood algorithm. Our aim was to evaluate the spatial resolution and sensitivity attainable with the seven-pinhole imaging device, together with the linearity for quantification on the tomographic images, and to test the instrument in obtaining tomographic images of different mouse organs. A spatial resolution better than $500 \mu \mathrm{m}$ and a sensitivity of $21.6 \mathrm{counts} \cdot \mathrm{s}^{-1} \cdot \mathrm{MBq}^{-1}$ were reached, as well as a correlation coefficient between activity and intensity better than 0.99 , when imaging ${ }^{99 m}$ Tc sources. Images of the thyroid, heart, lungs, and bones of mice were registered using ${ }^{99 \mathrm{~m}} \mathrm{Tc}$-labeled radiopharmaceuticals in times appropriate for routine preclinical experimentation of $<1 \mathrm{~h}$ per projection data set. Detailed experimental protocols and images of the aforementioned organs are shown. We plan to extend the instrument's field of view to fix larger animals and to combine data from both detectors to reduce the acquisition time or applied activity.
\end{abstract}

Key words: Tomography; Preclinical imaging; Molecular imaging; Mice

\section{Introduction}

In vivo imaging techniques, such as single photon emission computed tomography (SPECT) and positron emission tomography (PET), are becoming important tools in the preclinical environment, as appropriate instrumentation is becoming more accessible. Two main factors must be considered when imaging small animal organs: the instrument's spatial resolution and its sensitivity, both of which are directly related to the small size of the targets. Small animal organs are a factor of 300 (for rats) to 1000 (for mice) smaller than the equivalent organs of humans. Therefore, in order to have the same image quality as in clinical investigations, preclinical instruments must be built such that they can reach spatial resolutions better than clinical ones by roughly a factor of 10 . Sensitivity must also be improved, or, alternatively, larger activities or acquisition times must be considered during experiments. Most of the instruments recently developed for SPECT preclinical applications 
consider the use of pinhole collimators, given the implicit magnification factor, to overcome the limitation imposed by the detector's intrinsic spatial resolution, if the purpose is to upgrade clinical instruments (1-5). To obtain greater sensitivity, multipinhole collimators have been proposed, projecting individual images on independent areas of the detector, to minimize the overlap while making efficient use of the detector's area (6). In the case of specially designed instruments for preclinical applications, high intrinsic resolution scintillation detectors can be used, e.g., pixellated thallium-doped sodium iodide $[\mathrm{Nal}(\mathrm{TI})]$ or thallium-doped cesium iodide [CsI(TI)] columnar scintillators. In this situation, large magnification factors are no longer necessary, and it is even possible to consider demagnification to make better use of the available sensitive area (7). Sets of detector heads, each one with a single pinhole collimator, and arranged surrounding the target, are an alternative to improve sensitivity, as a large set of projections is acquired simultaneously, while each detector head guarantees improved spatial resolution (8).

The single photon emission microscope (SPEM), an instrument developed at the University of Illinois with the main purpose of making images of small structures inside the mouse brain, combines some of those technologies to reach high spatial resolution and appropriate sensitivity for preclinical research. The objective of this study was to evaluate the imaging capabilities of the instrument with in vivo animal targets. We have obtained images of different mouse organs, which allow us to conclude that, in its current configuration, the instrument can be used as the base of a core facility for molecular imaging preclinical applications. A preliminary description of the instrument's characteristics has been presented elsewhere (9).

\section{Material and Methods}

\section{SPEM}

The SPEM was developed by the University of Illinois at Urbana-Champaign, with the main objective of obtaining ultra-high spatial resolution SPECT images of small volumes inside the brains of mice (10). Currently, this equipment is installed at the Center for Preclinical Imaging of the CETEC/IIEPAE (Centro de Experimentação e Treinamento em Cirurgia, Instituto Israelita de Ensino e Pesquisa Albert Einstein) in São Paulo, Brazil. The SPEM consists of two independent imaging heads, which simultaneously acquire data from the target organ. Each imaging head is composed of a multipinhole collimator, a high intrinsic resolution columnar scintillator, an image intensifier and demagnifier tube, and a high-sensitivity electron-multiplying charge-coupling device (CCD). The main components of this instrument are shown in Figure 1. To create an experimental record, the target is located inside a transparent plastic tube or animal holder and

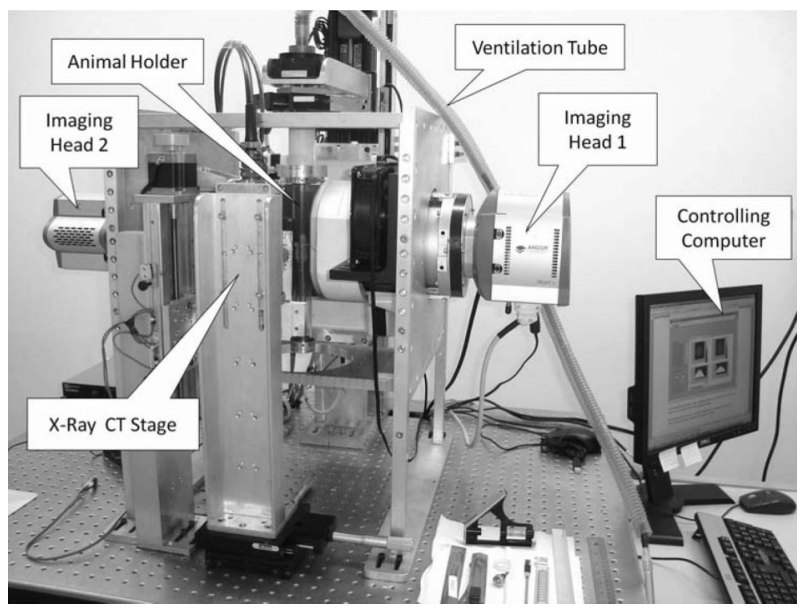

Figure 1. Single photon emission microscope (SPEM). The main components are labeled.

maintained in the vertical position to minimize oscillation of the internal organs during data acquisition. Oxygen can be supplied to the animal during the experiment, by means of a ventilation tube, which can also be used to provide inhaled anesthetics.

Planar projections of radiopharmaceutical distribution inside the target organ are obtained by means of multipinhole collimators: 19 and 7 pinholes, with 300and $450-\mu \mathrm{m}$ diameters for imaging heads 1 and 2 , respectively. The distribution, size, and quantity of pinholes are devised to minimize overlap of the individual projections and to maximize sensitivity and use of the detector's area. The projections are registered using a $\mathrm{CsI}(\mathrm{TI})$ columnar scintillator detector attached to an image intensifier and to a high-sensitivity CCD camera. Images are recorded following a photon-counting strategy, which consists of registering individual CCD frames at high speed, typically between 25 and 200 frames per second. In this way, individual frames contain just a few independent events of photon-detector interaction, superimposed on a background pattern. After recording the image, a preprocessing stage is carried out, when individual frames are evaluated to identify photon-detector interaction events. These events are assessed to determine subpixel interaction coordinates and deposited energy, which are saved in list-mode files. In a second preprocessing stage, the listmode files are read, and events are selected if the deposited energy is between appropriate limits corresponding to the radiopharmaceutical and are then organized to produce the set of projections $(11,12)$.

To produce the volumetric images, the projections are combined using a software tool specially developed for this application, based on the maximum likelihood expectation maximization algorithm $(13,14)$. As input, the software tool uses projection files and a model of the system's response to a point source. This system's 
response function is obtained by simulation, considering that a small size source is located along a large set of different positions in the instrument's field of view. The physical characteristics of the instrument, which are considered for calculating the response functions, are determined by acquiring a set of images of a real small size ${ }^{57}$ Co source on a short set of positions in the field of view (16 projections, 3 different radial positions, and 3 different vertical positions, for a total of 144 images).

The instrument's spatial resolution in the current configuration was determined by using a phantom composed of a set of 13 glass capillaries positioned next to each other. Seven of the 13 capillaries were filled with small quantities or sodium pertechnetate, interleaved with empty capillaries, resulting in variable distances between them. This phantom was imaged following our standard protocol (16 projections, 2 min/projection) with the sevenpinhole collimator imaging head. Sensitivity was assessed by imaging a small size source of sodium pertechnetate (46 MBq), positioned near the center of the field of view and on the rotation axis, for a period of $10 \mathrm{~min}$. From this record, the number of registered interaction events per unit time was determined. To verify the linearity between activity in the target and intensity in the reconstructed volumetric image, we used a set of five small-volume syringes containing different activities of sodium pertechnetate (from 7.75 to $59.0 \mathrm{MBq}$ ) in the same volume $(0.2 \mathrm{~mL})$. After processing of the images, we defined a cylindrical region of interest $3 \mathrm{~mm}$ in diameter and $10 \mathrm{~mm}$ in length to determine the mean intensity value. Those data were compared to determine the Pearson correlation coefficient.

\section{Animals}

In order to test the imaging capabilities of the SPEM, young and adult Swiss mice, varying from 30 to $50 \mathrm{~g}$, were included in the experiments. All animals were maintained under controlled temperature and humidity conditions $\left(22 \pm 2^{\circ} \mathrm{C}, 50 \%\right.$ relative humidity), with free access to food and water. Special care was taken to avoid unnecessary suffering to the animals. All the experimental protocols were evaluated and approved by the
Institutional Animal Care and Use Committee.

\section{SPECT imaging protocol}

In this experiment, animals were injected with appropriate radiopharmaceuticals targeted for specific organs. Even though SPEM development was aimed at imaging brain substructures, we assessed the instrument's imaging capabilities focusing on different organs: thyroid, lungs, heart, and bones, labeled with sodium pertechnetate, ${ }^{99 \mathrm{~m}} \mathrm{Tc}-\mathrm{MAA},{ }^{99 \mathrm{~m}} \mathrm{Tc}-\mathrm{MIBI}$, and ${ }^{99 \mathrm{~m}} \mathrm{Tc}-\mathrm{MDP}$, respectively. In all cases, different injected activities and times between injection of the radiopharmaceutical and the beginning of the image acquisition were tested, in order to determine those that produce the best quality tomographic images. Other parameters, including number of projections, time per projection, and number and distribution of pinholes on the collimator, were maintained constant for each target organ. The experimental parameters corresponding to the results described in this study are shown in Table 1.

To prepare for image acquisition, animals were anesthetized by inhalation with isofluorane and the radiopharmaceutical was injected through the lateral tail vein. After the appropriate uptake and background washout time, the animals were anesthetized intraperitoneally with a combination of ketamine and xylazine $(125: 12.5 \mathrm{mg} / \mathrm{kg})$ and placed inside the animal holder The animals remained in the vertical position during the data acquisition protocol, in order to minimize oscillation of the internal organs because of the rotation. Neither heart rate nor body temperature was monitored during the experiment. However, all the animals survived the imaging experiments.

Tomographic reconstruction was carried out using a specially developed software tool based on the iterative maximum likelihood expectation maximization algorithm. After each iteration, a Gaussian smoothing filter was applied, whose width varied from five to one pixels, one pixel for every 20 iterations, for a total of 100 iterations. Visual inspection of the resulting images allowed us to verify that, after iteration 80 , the images became very noisy. For that reason, iteration 80 was considered the final one for all the imaging tests, unless otherwise stated.

Table 1. Experimental parameters for imaging with SPEM: 16 projections and the seven-pinhole collimator imaging heads were used.

\begin{tabular}{|c|c|c|c|c|}
\hline Organ & Radiopharmaceutical & Activity (MBq) & $\Delta \mathrm{t}(\min )$ & $\begin{array}{l}\text { Time per projection } \\
(\mathrm{min})\end{array}$ \\
\hline Thyroid & Sodium pertecnetate & 74 & 5 & 3 \\
\hline Lungs & MacroAggregated albumin- ${ }^{99 m} \mathrm{Tc}(\mathrm{MAA})$ & 185 & 5 & 3 \\
\hline Heart & Sestamibi- ${ }^{99 \mathrm{~m}} \mathrm{Tc}(\mathrm{MIBI})$ & 74 & 60 & 5 \\
\hline Bones & Methylene diphosphonate- ${ }^{99 \mathrm{~m}} \mathrm{Tc}$ (MDP) & 185 & 60 & 3 \\
\hline
\end{tabular}

$\Delta t$ corresponds to the time between the injection of the radiopharmaceutical and the beginning of the recording. SPEM: single photon emission microscope. 

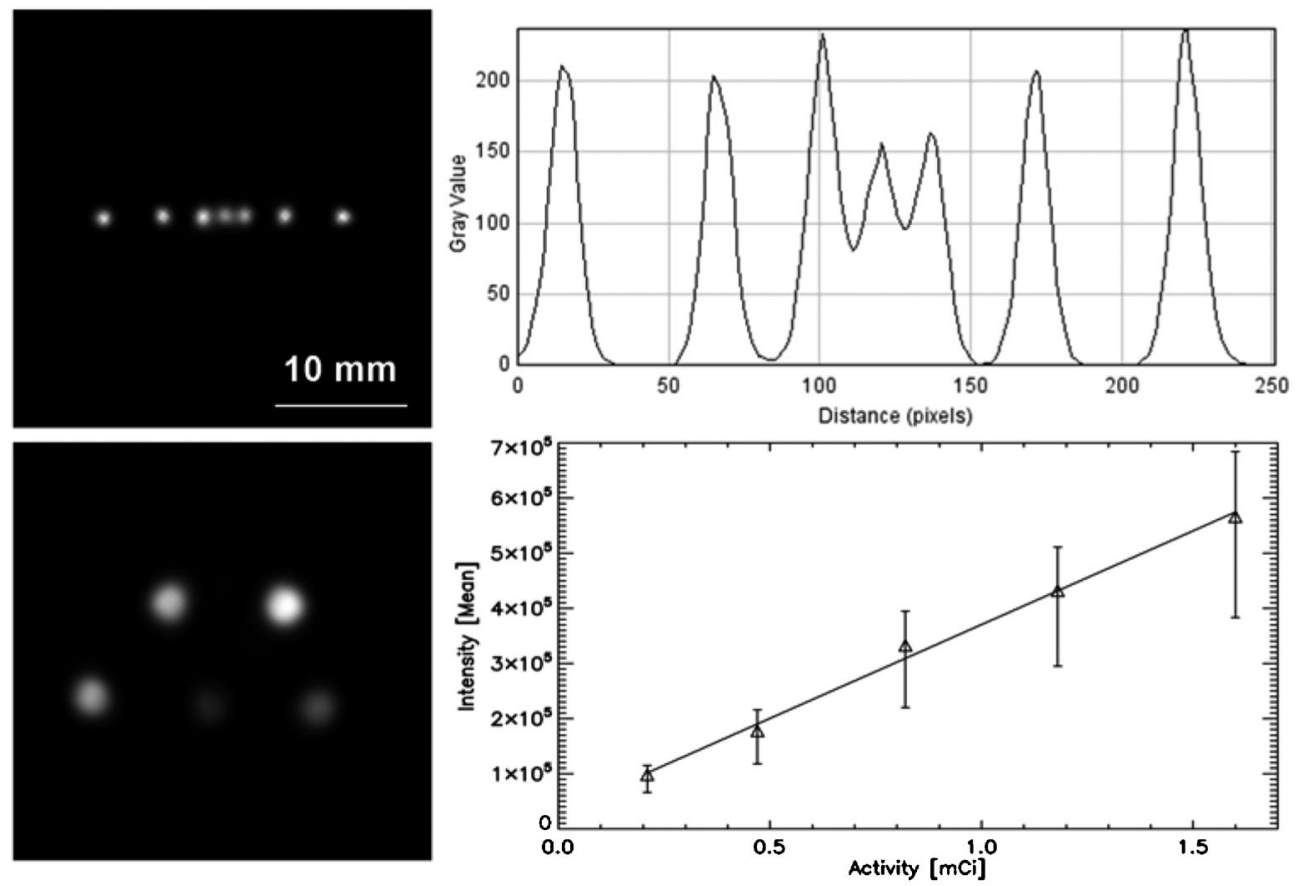

Figure 2. Spatial resolution and quantification. Upper panels, capillary tube phantom. Left: characteristic slice through the volumetric reconstruction of the phantom's emission. Right: intensity profile along a line intersecting the seven capillaries. It can be seen that all the capillary's emissions are well identified. Lower panels, quantification phantom. Left: characteristic slice through the volumetric reconstruction of the phantom's emission. Right: plot of the activity in the phantom versus mean intensity in the phantom.

\section{Results}

\section{Spatial resolution, sensitivity, and quantification linearity verification}

Figure 2 (upper panels) shows the results of the spatial resolution determination test. At the left, a slice through the volumetric reconstruction of the phantom's emission can be seen. All the filled capillaries are well identified, even those that are next to one another, at the center of the phantom. Considering that those capillaries have $1.5-\mathrm{mm}$ external diameter and $1.0-\mathrm{mm}$ internal diameter, the glass space between two contiguous tubes is $0.5 \mathrm{~mm}$ thick, so we can conclude that sources located at distances of roughly $500 \mu \mathrm{m}$ can be individually identified. An intensity profile along the center of the capillaries is shown in the right panel of the same figure. Again, clear identification of the three central sources can be verified. Additionally, by fitting a Gauss function to the individual line profiles, we found a mean full width at half maximum (FWHM) of $1.06 \mathrm{~mm}$. Removing the effect of the capillary's internal diameter of $1 \mathrm{~mm}$, that value implies a system's spatial resolution of $0.35 \mathrm{~mm}$ (FWHM). For the sensitivity, we found that 591,000 events were registered in a period of $10 \mathrm{~min}$, which results in a total of 21.6 counts $\cdot \mathrm{s}^{-1} \cdot \mathrm{MBq}^{-1}$. Finally, in
Figure 2 (lower panels), we illustrate the results of imaging the quantification phantom. By comparing the activities in the phantom with the mean intensities measured from the reconstructed image, we found a Pearson correlation coefficient greater than 0.99 .

\section{Imaging of mouse organs with ${ }^{99 \mathrm{~m}} \mathrm{Tc}$-labeled radiopharmaceuticals}

In Figures 3 to 6 , we illustrate the results of imaging different mouse organs with specific radiopharmaceuticals, all labeled with ${ }^{99 \mathrm{~m}} \mathrm{Tc}$. In the upper panels of Figure 3 , we show 4 of the 16 projections obtained while imaging the mouse thyroid gland, labeled with sodium pertechnetate. Besides the thyroid, a larger structure corresponding to the salivary glands is visualized. In the lower panels, we show characteristic slices through the volumetric reconstruction of the emission from the thyroid region, where it is possible to verify that both lobes of the thyroid are clearly identified, which allows us to define a practical value for the spatial resolution when imaging in vivo biological targets. Considering a lobe diameter and an inter-lobe distance of 0.8 and $1.2 \mathrm{~mm}$, respectively, we can verify that in vivo sources separated by nearly $1 \mathrm{~mm}$ between them can be individually identified. Similarly, in Figure 4, we show a set of three slices perpendicular to 

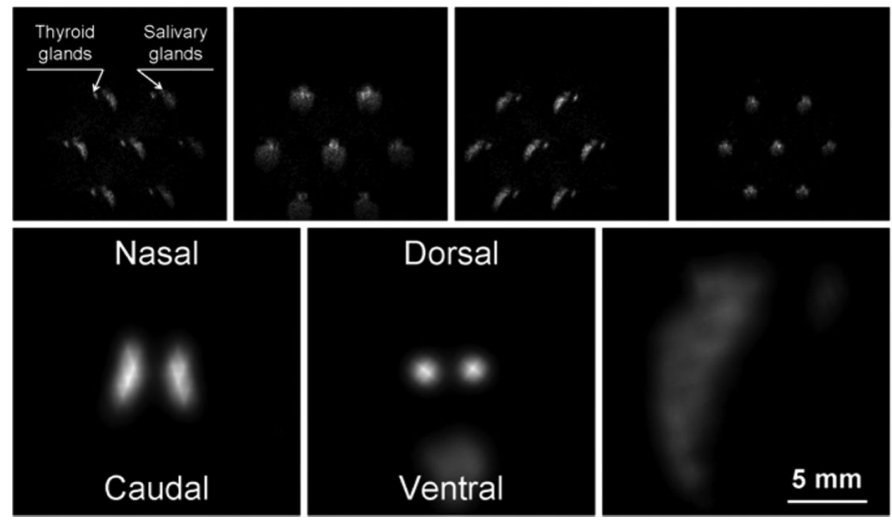

$100 \%$
Figure 3. Thyroid images. Upper panels, sequence of 4 of the 16 projections obtained with the seven-pinhole imaging device of the mouse neck area, labeled with sodium pertechnetate. Lower panels, characteristic slices through the reconstructed volumetric model of the radioactive emission from the mouse thyroid and salivary glands along the three main axes, after 80 iterations. the long axis of the volumetric reconstruction of the emission from the mouse heart, labeled with ${ }^{99 m} \mathrm{Tc}-\mathrm{MIBI}$. This is a challenging organ to image, mainly because it is beating fast, but also because of the emission contribution of neighboring organs, which also cumulate the same radiopharmaceutical, e.g., the liver and kidneys.

Extended structures can also be imaged with SPEM, as in Figure 5, which shows, in the upper panels, 4 of the 16 projections obtained while imaging the mouse lungs, labeled with ${ }^{99 m}$ Tc-MAA. In this case, the individual pinhole projections almost filled the complete field of view, which imposes a limit on the size of the target. The lower panels show two characteristic slices through the volumetric reconstruction. Other examples are given in Figure 6, which again shows a set of 4 of the 16 projections obtained while imaging the mouse skull, labeled with ${ }^{99 \mathrm{~m}} \mathrm{Tc}-\mathrm{MDP}$, together with two representative slices through the volumetric reconstruction.

\section{Discussion}

In the last two decades, a great effort has been made to transfer clinical imaging techniques to the preclinical environment. There is almost unanimous agreement that the use of imaging techniques allows for a significant increase in the statistical value of the experimental data obtained while the animal remains alive. This is mainly because the animal can be used as its own control, instead of comparing experimental groups where all the experimental variables cannot always be controlled, and can be evaluated at different time points during the experiment (15). At the same time, the use of these techniques reduces maintenance costs because fewer animals are needed, which also has clear ethical benefits.

With this objective, two main lines of development have been pursued: upgrading already available clinical gammacameras to be used during the time they are available, or developing specific-purpose instruments, dedicated to imaging small animals. Following the first approach, mechanical adaptations include the use of single or multiple pinhole collimators, most often in association with a computer-controlled system for positioning and rotation of the animal in front of the collimator $(3,4,16-18)$. In order to avoid movement of the target, which can imply oscillation of the internal organs, the use of a set of detectors mounted on a ring, completely surrounding the target, has been proposed, which results in special-purpose instruments based on large scintillation detectors, for example, FastSPECT II (8), nanoSPECT (19), and U-SPECT II (20). The second approach is based on the implementation of sets of small-size, high-resolution imagers, focusing on small volume targets like mice or young rats. Examples of this kind of instrument are FastSPECT III (21) and SPEM. In both cases, highresolution columnar scintillators are used as gamma-ray detectors, whose emission is amplified by image intensifiers and detected by CCD cameras. Unlike SPEM, FastSPECT III consists of 20 single pinhole collimator imagers, mounted on three rings surrounding the target space. As already described, SPEM uses only two multipinhole collimator imagers, and the target must be rotated for recording.
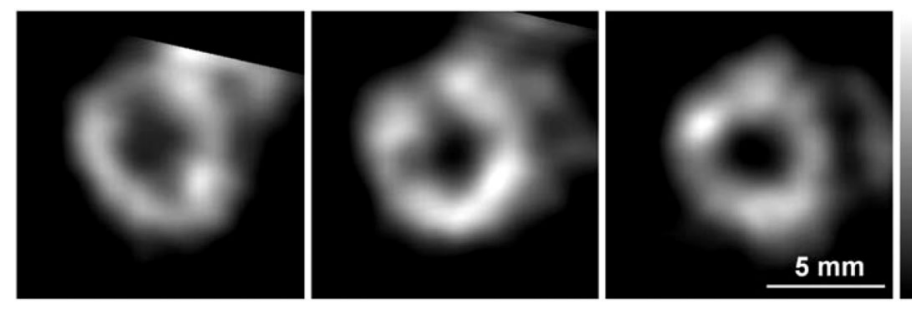

$100 \%$

Figure 4. Cardiac images. Characteristic slices perpendicular to the main axis of the reconstructed volumetric model of the radioactive emission from the mouse heart, labeled with ${ }^{99 \mathrm{~m}} \mathrm{Tc}$-MIBI, after 80 iterations. 

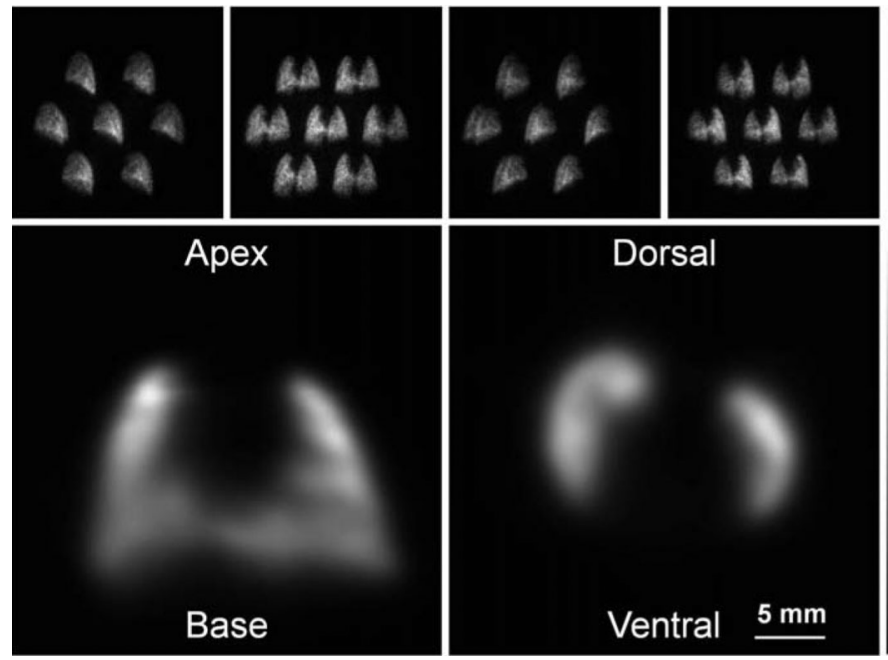

Figure 5. Pulmonary images. Upper panels, sequence of 4 of the 16 projections obtained with the seven-pinhole imaging device of the mouse chest area, labeled with ${ }^{99 m}$ Tc-MAA. Lower panels, characteristic transversal and coronal slices through the reconstructed volumetric model of the radioactive emission from the mouse lungs, after 60 iterations.
While developing these instruments, special attention has been given to spatial resolution. Considering the small size of the animals under scrutiny and, consequently, of the organs and structures of interest, the ability to identify neighboring sources of small size is crucial. Spatial resolution can be improved in several ways: 1) by locating the target as near to the collimator as possible, which increases the magnification, provided that the projections are not truncated as the animal is rotated; 2) by labeling the imaging pharmaceuticals with low-energy gamma emitters, e.g., ${ }^{125}$ iodine; 3 ) by reducing the diameter of the pinhole collimator. However, sensitivity is also a very important parameter. When considering the mean life of ${ }^{99 \mathrm{~m}} \mathrm{Tc}$, the most common gamma emitter in nuclear medicine, and the biological mean life of the radiopharmaceutical in the animal, experimental time is limited to no more than a couple of hours. Additionally, depending on the anesthetics, another limit is imposed of not much more than roughly $1 \mathrm{~h}$. And finally, if routine sets of experiments are to be carried out, a value of around 30 to $60 \mathrm{~min}$ for data acquisition, or even better, is optimal. As sensitivity is mostly limited by the diameter of the collimator, alternatives to recover it include the use of large sets of imagers with single pinhole collimators or the use of multipinhole collimators, taking special care to avoid or limit the overlapping of individual projections on each detector.

In the case of SPEM, very small diameter pinholes, in combination with high intrinsic spatial resolution detectors, are used to guarantee that small structures inside the mouse body, roughly $350 \mu \mathrm{m}$ from one to the other, can be individually identified. Sensitivity is recovered by using large sets of pinholes (7 and 19), whose opening angle limits the size of the field of view, thus minimizing the overlap of individual images. However, acquisition times are of the order of 45 to $80 \mathrm{~min}$, which is already large for
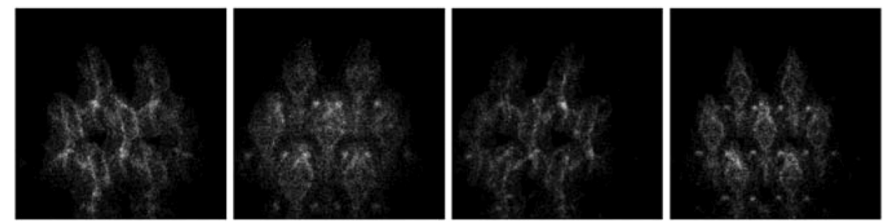
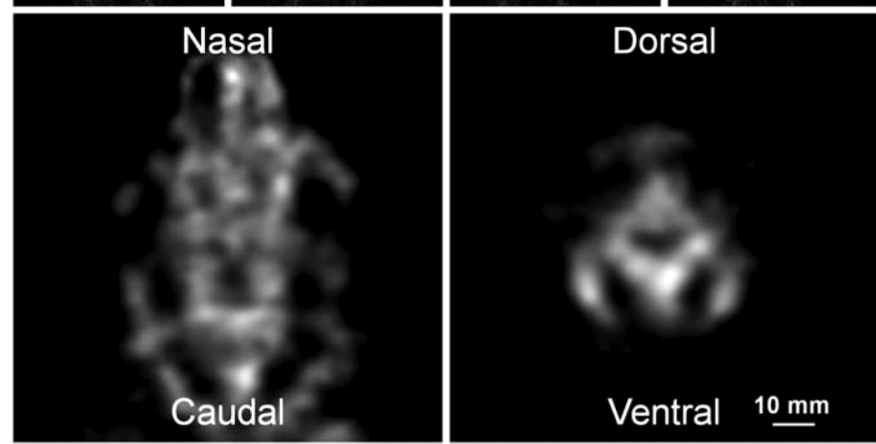

$100 \%$

www.bjournal.com.br
Figure 6. Bone images. Upper panels, sequence of 4 of the 16 projections obtained with the sevenpinhole imaging device of the mouse head area, labeled with ${ }^{99 \mathrm{~m}} \mathrm{Tc}-\mathrm{MDP}$. Lower panels, characteristic transversal and coronal slices through the reconstructed volumetric model of the radioactive emission from the mouse skull, after 80 iterations. 
some experimental protocols. For this reason, strategies are being considered to combine the acquired data from both detectors to reduce the acquisition time by a factor of 2 , and also to duplicate the system to include at least four detection cameras, again reducing the acquisition time by an additional factor of 2 . In any case, as illustrated in this study, the instrument allows us to obtain images of several different mouse organs, so it can be used in experimental protocols in many preclinical research areas such as cardiology, nephrology, oncology, or the respiratory system. On the other hand, with small modifications,

\section{References}

1. Beekman F, van der Have F. The pinhole: gateway to ultrahigh-resolution three-dimensional radionuclide imaging. Eur J Nucl Med Mol Imaging 2007; 34: 151-161, doi: 10.1007/ s00259-006-0248-6.

2. Golestani R, Wu C, Tio RA, Zeebregts $C J$, Petrov $A D$, Beekman FJ, et al. Small-animal SPECT and SPECT/CT: application in cardiovascular research. Eur J Nucl Med Mol Imaging 2010; 37: 1766-1777, doi: 10.1007/s00259-0091321-8.

3. Mejia J, Galvis-Alonso OY, Castro AA, Braga J, Leite JP, Simoes MV. A clinical gamma camera-based pinhole collimated system for high resolution small animal SPECT imaging. Braz J Med Biol Res 2010; 43: 1160-1166, doi: 10.1590/S0100-879X2010007500128.

4. Difilippo FP. Design and performance of a multi-pinhole collimation device for small animal imaging with clinical SPECT and SPECT-CT scanners. Phys Med Biol 2008; 53: 4185-4201, doi: 10.1088/0031-9155/53/15/012.

5. Wagenaar DJ, Engdahl JC, Simcic V, Hawman EG, Mertelmeier T, Mahmood $U$, et al. Use of conventional gamma cameras for small animal imaging. IEEE Nucl Sci Symp Conf Rec 2000; 86-90.

6. Branderhorst W, Vastenhouw B, van der Have F, Blezer EL, Bleeker WK, Beekman FJ. Targeted multi-pinhole SPECT. Eur J Nucl Med Mol Imaging 2011; 38: 552-561, doi: 10. 1007/s00259-010-1637-4.

7. Barrett HH, Hunter WCJ. Detectors for small-animal SPECT I. In: Kupinsky MA, Barrett HH (Editors), Small-animal SPECT imaging. Boston: Kluwer Academic Publishers; 2013. p 9-48.

8. Furenlid LR, Wilson DW, Chen YC, Kim H, Pietraski PJ, Crawford MJ, et al. FastSPECT II: A Second-Generation High-Resolution Dynamic SPECT Imager. IEEE Trans Nucl Sci 2004; 51: 631-635, doi: 10.1109/TNS.2004.830975.

9. Reis MA, Mejia J, Batista IR, Barboza MR, Nogueira SA, Wagner J, et al. SPEM: a state-of-the-art instrument for high resolution molecular imaging of small animal organs. Einstein 2012; 10: 209-215, doi: 10.1590/S1679-450820120 00200015

10. Li N, Meng L-J. Adaptive angular sampling for SPECT imaging. IEEE Trans Nucl Sci 2011; 58: 2205-2218, doi: 10. 1109/TNS.2011.2164935.

11. Meng L-J. An intensified EMCCD camera for low energy gamma ray imaging applications. IEEE Trans Nucl Sci larger animals can also be studied, such as hamsters, gerbils, or young rats, to increase the range of experimental possibilities.

\section{Acknowledgments}

Research supported by Associação Beneficente Alzira Denise Hertzog da Silva (ABADHS) and by FAPESP (\#2006/61333-3). M.A. Reis is supported by FAPESP (\#2008/08902-5).

2013; 53: 2376-2384, doi: 10.1109/TNS.2006.878574.

12. Meng L-J, Fu G. Investigation of the intrinsic spatial resolution of an intensified EMCCD scintillation camera. IEEE Trans Nucl Sci 2008; 55: 2508-2517, doi: 10.1109/ TNS.2008.2004278.

13. Shepp LA, Vardi Y. Maximum likelihood reconstruction for emission tomography. IEEE Trans Med Imaging 1982; 1: 113-122, doi: 10.1109/TMI.1982.4307558.

14. Lange $\mathrm{K}$, Carson R. EM reconstruction algorithms for emission and transmission tomography. J Comput Assist Tomogr 1984; 8: 306-316.

15. Meikle SR, Kench P, Kassiou M, Banati RB. Small animal SPECT and its place in the matrix of molecular imaging technologies. Phys Med Biol 2005; 50: R45-R61, doi: 10.1088/0031-9155/50/22/R01.

16. Wu MC, Gao DW, Sievers RE, Lee RJ, Hasegawa BH, Dae MW. Pinhole single-photon emission computed tomography for myocardial perfusion imaging of mice. J Am Coll Cardiol 2003; 42: 576-582, doi: 10.1016/S0735-1097(03)00716-2.

17. Meikle SR, Kench P, Weisenberger AG, Wojcik R, Smith MF, Majewski S, et al. A prototype coded aperture detector for small animal SPECT. IEEE Trans Nucl Sci 2002; 49 2167-2171, doi: 10.1109/TNS.2002.803802.

18. Accorsi R, Gasparini F, Lanza RC. A coded aperture for high-resolution nuclear medicine planar imaging with a conventional Anger camera: experimental results. IEEE Trans Nucl Sci 2001; 48: 2411-2417, doi: 10.1109/23.983 251

19. Schramm NU, Lackas C, Hoppin JW, Forrer F, de Jong M. The nanoSPECT/CT: a high-sensitivity small-animal SPECT/CT with submillimeter spatial resolution. Eur $J$ Nucl Med Mol Imaging 2006; 33: S117, doi: 10.1007/ s00259-005-1899-4.

20. van der Have F, Vastenhouw B, Ramakers RM, Branderhorst W, Krah JO, Ji C, et al. U-SPECT-II: An ultra-high-resolution device for molecular small-animal imaging. J Nucl Med 2009; 50: 599-605, doi: 10.2967/ jnumed.108.056606.

21. Miller BW, Furenlid LR, Moore SK, Barber HB, Nagarkar VV, Barrett HH. System Integration of FastSPECT III, a Dedicated SPECT Rodent-Brain Imager Based on BazookaSPECT Detector Technology. IEEE Nucl Sci Symp Conf Rec (1997) 2009; Oct. 24 2009-Nov. 1 2009: 4004-4008. 